\title{
Determination of Oxidative Energy Metabolism and Plasma LDH Isoenzyme Patterns of Dolphins
}

\author{
Yasuko Hirakawa ${ }^{1}$, Koh Kawasumi $^{1}$, Peter Lee ${ }^{1}$, Nobuko Mori ${ }^{1}$, Ichiro Yamamoto ${ }^{1}$, \\ Fumio Terasawa ${ }^{2}$ and Toshiro Arai ${ }^{*}, 1$
}

\author{
${ }^{1}$ Department of Veterinary Science, School of Veterinary Medicine, Nippon Veterinary and Life Science University, 1-7- \\ 1 Kyonancho, Musashino, Tokyo 180-8602, Japan \\ ${ }^{2}$ Enoshima Aquarium, 2-19-1 Katase-kaigan, Fujisawa, Kanagawa 251-0035, Japan
}

\begin{abstract}
In this study, oxidative energy metabolism was determined in 5 captive bottlenose dolphins (Tursiops truncatus), and subsequently compared to 6 Thoroughbred riding horses, and 12 lactating Holstein cows in order to assess symmorphosis of each animal species. Plasma metabolites profile, plasma MDH (M) and LDH (L) activities, M/L ratio, and plasma LDH isoenzyme distribution patterns were all examined. Overall, dolphins appear to have the greatest level of oxidative energy metabolism amongst horses and cows, due to having the greatest levels of plasma MDH activity. In addition, dolphin energy production/usage efficiency, via oxidative metabolism, was considered to be second $(\mathrm{M} / \mathrm{L}$ ratio = 0.49 ) behind that of horses $(\mathrm{M} / \mathrm{L}$ ratio $=0.79)$, possibly due to an increased reliance of non-oxidative energy metabolism over horses. In spite of demonstrating the lowest oxidative energy production/usage efficiency $(\mathrm{M} / \mathrm{L}$ ratio $=0.15)$, cows also demonstrated the highest plasma LDH activity amongst all animal species. Although all animal groups displayed differing plasma LDH isoenzyme distribution patterns, dolphins and horses demonstrated a similarity with LDH-3 isoenzyme predominating in plasma; whereas LDH-1 isoenzyme predominates in cow plasma, thus inferring differences in oxidative/non-oxidative metabolism for energy generation/usage. Therefore, plasma MDH and LDH activity levels, $\mathrm{M} / \mathrm{L}$ ratio, and plasma $\mathrm{LDH}$ isoenzyme pattern can all be useful indicators for better understanding oxidative energy metabolism and monitoring of captive animals' health. As it is not easy to obtain tissue samples from animals, the development of blood indicators for evaluating whole body metabolic state is necessary.
\end{abstract}

Keywords: Cow, dolphin, horse, LDH isoenzyme, malate dehydrogenase.

\section{INTRODUCTION}

Monitoring and understanding the relevance of hematological data is crucial for health management of animals, especially those bred in captivity. Since little is known about the health conditions of marine mammals, it is very difficult to determine disease development, until it is in well advanced stages. A better understanding of metabolism patterns can lead to more efficient management and disease prevention strategies of these animals. Since blood collection can be easily performed, during routine health examinations, plasma metabolite values can serve as a useful index for early detection of diseases. With dolphins, it is rather difficult to detect health abnormalities, especially in the early stages, owing to the fact that standard normal plasma metabolite value ranges have yet to be established and little is understood thus far. Moreover, information about dolphin energy metabolism is scant so further developments will be of use to better understand basic dolphin basal metabolism.

Increased enzyme activity within the malate-aspartate shuttle appears to produce efficient energy metabolism in animal tissues [1], with malate-aspartate shuttle activity

*Address correspondence to this author at the Department of Veterinary Science, School of Veterinary Medicine, Nippon Veterinary and Life Science University, 1-7-1 Kyonancho, Musashino, Tokyo 180-8602, Japan; Tel: 81-422-31-4151; Fax: 81-422-31-7841; E-mail: tarai@nvlu.ac.jp modulating according to variations in energy metabolism needs [2]. Malate dehydrogenase (MDH) is necessary for gluconeogenesis and lipogenesis, and plays a significant role in the malate-aspartate shuttle [3], transporting cytosolic NADH into mitochondria followed by oxidative ATP production [4]. Alternately, lactate dehydrogenase (LDH) converts pyruvate to lactate consuming cytosolic NADH. Glucose is thought to be metabolized to pyruvate, in cytosolic glycolysis, and is the main energy source in most mammals [5]. Each animal species has specific values for plasma and leukocyte MDH, LDH enzyme activities, and $\mathrm{MDH} / \mathrm{LDH}$ activity (M/L) ratio [6]. Furthermore, Washizu et al. [7] reported that cytosolic $\mathrm{M} / \mathrm{L}$ ratio is considered to be a useful indicator to evaluate metabolic states in animal tissues.

"Symmorphosis" as originally proposed by Taylor and Weibel [8] states that biological design will be optimized, such that each structural element in a functional chain matches the maximal requirements of the overall functional system [9]. The concept was motivated by the authors' beliefs that animals are built reasonably and their conviction that structural design is optimized because maintaining biological structures with their often high turnover rates is costly [10]. For an initial empirical test of symmorphosis, Weibel and Taylor focused on the mammalian respiratory system and proposed two comparative tests. First, for 
animals differing in size, the scaling of respiratory structures should parallel the scaling of maximal oxygen consumption. Second, for animals of similar size, differences in structural capacities should match differences in $\mathrm{VO}_{2} \max$, the maximal rate of $\mathrm{O}_{2}$ consumption attained during exercise of progressively increasing intensity. $\mathrm{VO}_{2}$ max sets an upper limit to the intensity of work that can be sustained aerobically for prolonged periods, and hence should be of considerable selective importance. As such, different species should reflect optimization and differences in oxidative energy metabolism, which would be reflected in muscle fiber (aerobic versus anaerobic) and metabolic profile differences between species.

Therefore, the aim of the present study was twofold. First, we sought to determine plasma metabolite profiles of healthy captive dolphins, and assess their energy metabolism state by examining plasma $\mathrm{MDH}$ and $\mathrm{LDH}$ activities, M/L ratio, and $\mathrm{LDH}$ isoenzyme patterns since such information is lacking in the literature. Second, we sought to compare the aforementioned parameters between healthy captive bottlenose dolphins and thoroughbred riding horses and lactating Holstein cows, in order to assess "Symmorphosis" regarding oxidative energy metabolism. Horses and cows may serve as practical comparisons for the following reasons. First, horses and cows are similarly large animals as dolphins, and have also been trained or domesticated by humans, as well. Second, because dolphins normally have relatively good motility, horses and cows can serve as good comparisons because horses are also quite active, whereas cows exhibit little physical activity; thereby highlighting differences in energy usage between all 3 species. Third, there is evidence demonstrating that dolphins may be closely related to even-toed ungulates, such as cows, pigs and camels [11]. Lastly, our laboratory has previously reported on energy metabolism of horses and cows $[6,12,13]$.

\section{MATERIALS AND METHODS}

\section{Animals}

Five captive bottlenose dolphins (Tursiops truncates), 6 Thoroughbred riding horses, and 12 lactating Holstein cows were included in this study. All animals were diagnosed to be healthy and exhibited no clinical signs for disease. The bottlenose dolphins were part of the population at Enoshima Aquarium in Kanagawa, Japan. The tank size, seawater volume, air and water temperatures of the dolphin pool at Enoshima Aquarium have been previously presented [30]. Thoroughbred riding horses were maintained and trained in our university. The lactating Holstein cows were maintained at Koizumi Milk Farm in Tokyo, Japan. Dolphins were fed 4 to 8 times per day, between 9:00 and 17:30, and consumed approximately 10 to $14 \mathrm{~kg}$ daily. Horses were fed a daily diet of $\sim 5 \mathrm{~kg}$ in total, 3 times per day, between 6:00 and 16:00. Cows were given 29 to $32 \mathrm{~kg}$ of feed daily, administered 4 times daily between 5:30 and 10:00. All animals involved in this study lived in captivity.

Regarding physical activity, in general, dolphins at the Enoshima Aquarium were active as compared to other animal groups, constantly moving in and around the tank, due to training or shows in the daytime. Alternatively, horses at the university were usually immobile during the day, standing still, except for their daily exercise regiment, lasting
1 to 2 hours daily. Lastly, cows at the Koizumi Milk Farm were immobile, usually standing still. The cows were able to supply approximately $30 \mathrm{~mL}$ of milk per day. Approval for this work has been given by the Nippon Veterinary and Life Science University Animal Research Committee.

\section{Sample Collection}

Blood sampling from dolphins was conducted on a monthly basis, as a part of their routine physical exams at Enoshima Aquarium. Briefly, blood was taken from the tail fluke of dolphins by venipuncture, using a sterile 21 gauge disposable butterfly needle. Because the dolphins were trained for blood sampling and/or other routine physical exams, they can voluntarily display their tail or body when samples need to be collected. All dolphin blood samples were obtained 16 to 17 hours after their last feeding. Blood sampling from horses and cows was obtained from the jugular or caudal vein, respectively, early in the morning (5:00-7:00 am). The animals showed no excitement or fear, when blood sampling was conducted, since they were accustomed to blood sampling. Blood samples from all animals were centrifuged at $3500 \mathrm{rpm}$ for $10 \mathrm{~min}$ at $4{ }^{\circ} \mathrm{C}$ in order to obtain plasma samples, which were subsequently frozen at $-80^{\circ} \mathrm{C}$ until further use.

\section{Plasma Metabolite Assays}

Plasma biochemistry analysis on alanine aminotransferase (ALT), alkalanine phosphatase (ALP), aspartate aminotransferase (AST), blood urea nitrogen (BUN), creatinine, glucose, lactate dehydrogenase (LDH), total cholesterol (TCho), total protein (TP), and triglycerides (TG) was performed using an AU680 auto analyzer (Beckman Coulter, CA, USA) with the manufacturer's reagents. Free fatty acid (FFA) concentration was measured using a Wako NEFA-C test commercial kit (Wako Pure Chemical Industries, Inc., Tokyo, Japan).

\section{Enzyme Activity Assays and LDH Isoenzyme Profiling}

The activities of malate dehydrogenase (MDH) and lactate dehydrogenase (LDH) in plasma were measured as previously described (LDH [31] and MDH [32]). All enzymatic activities were measured between $24-26{ }^{\circ} \mathrm{C}$ and expressed as $U$ per liter of plasma. The enzyme unit (U) was defined as $1 \mu \mathrm{mol}$ of substrate degraded per min. The $\mathrm{M} / \mathrm{L}$ ratio was calculated as $\mathrm{MDH}$ activity divided by $\mathrm{LDH}$ activity. Plasma LDH isoenzyme patterns were detected by the biphasic agarose gel electrophoresis method utilizing commercial Quickgel LD gels (Helena Laboratories, Saitama, Japan). In brief, $30 \mu 1$ plasma sample volumes were loaded into the dipping well of an Epalyzer 2 Electrophoresis Processing Analyzer, of which 5-6 $\mu$ l was loaded onto the gel, and run with a $13 \mathrm{~min}$ set migration time at $240 \mathrm{~V}$ and at $15{ }^{\circ} \mathrm{C}$. After migration, the gels underwent a 14 min reaction time, followed by a $12 \mathrm{~min}$ and $30 \mathrm{~s}$ decolorizing and fixing time, respectively. LDH fractions were assessed and analyzed using Edbank III analysis software (Helena Laboratories).

\section{Statistical Analysis}

Results are expressed as means $\pm \mathrm{SD}$. Paired groups were compared using the Mann-Whitney U-test for data with nonnormal distribution, and statistical significance was set at 
$P<0.05$. All tests were performed using Sigmaplot analysis software (Sigmaplot 11.0, Build 11.0.077; Systat Software Inc., San Jose, CA).

\section{RESULTS}

\section{Plasma Metabolite Assays}

All values for the various measured plasma metabolites, for all animal groups, are shown in Table 1. There was no significant difference in glucose levels between dolphins and horses; however dolphin glucose level was significantly higher as compared to that of cows (Mann Whitney U-test, $\mathrm{p}<0.05)$. Dolphins also displayed a significantly higher level of TG, than either horses $(p=0.019)$ or cows $(p=0.001)$. TCho levels in dolphins and cows were both significantly higher than that of horses $(\mathrm{p}=0.002)$. FFA in dolphins was also significantly higher than that of either cows $(p=0.004)$ or horses $(p=0.002)$. Lastly, dolphins had a significantly higher level of ALP as compared to either cows $(p=0.001)$ or horses $(\mathrm{p}=0.002)$.

\section{MDH, LDH, and M/L Ratio}

A comparison of plasma MDH and LDH activities, in addition to $\mathrm{M} / \mathrm{L}$ ratio between all 3 animal groups is displayed in Table 2. Although no significant difference in MDH activity was noted between any of the groups, LDH activity in dolphins was significantly (Mann Whitney U-test, $\mathrm{p}<0.05)$ higher than that of horses $(\sim 2$ fold $)$, and lower than that of cows $(\sim 65 \%$ lower), respectively. As a result, $\mathrm{M} / \mathrm{L}$ ratio in each animal species was significantly different from one another (Mann Whitney U-test, $\mathrm{p}<0.05$ ). Horses demonstrated the highest $\mathrm{M} / \mathrm{L}$ ratio (0.79), followed by dolphins (0.49) and cows (0.15), respectively.

\section{LDH Isoenzyme Patterns}

LDH isoenzyme patterns for all animal groups are presented in Fig. (1).

Although all animal groups displayed significant differences in isoenzyme pattern distribution, dolphins and horses demonstrated a similarity in LDH isoenzyme pattern with LDH-3 predominating, and LDH-2 and -3 making up $\geq 50 \%$ of total plasma LDH, in both species (Table 3). The isoenzyme pattern in dolphins, in descending \% amount of total LDH, was LDH-3 (39.62 $\pm 1.07 \%)$, LDH-2 (29.56 $1.43 \%$ ), LDH-4 (17.18 $\pm 1.78 \%$ ), and LDH-1 (10.46 $\pm 0.97 \%)$. Alternately, the isoenyme pattern in horses, in descending amount, was LDH-3 (31.55 $\pm 2.20 \%)$, LDH-1 (29.88 $\pm 3.02 \%)$, LDH-2 (25.30 \pm

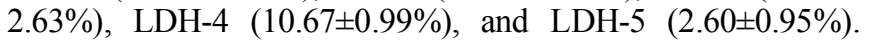
Unlike both dolphins and horse, LDH-1 (47.07 $1.56 \%)$ was the most dominant isoform in cows, followed by LDH-2 (27.77 \pm 0.78$)$, LDH-3 (15.45 \pm 1.11$)$, LDH-4 (5.60 \pm 0.50$)$, and LDH-5 (4.12 \pm 0.78$)$.

\section{DISCUSSION}

"Symmorphosis" as originally proposed by Taylor and Weibel [8] dictates that there should be a symbiosis between form and function for every animal species. In general, dolphins are constantly moving, hence their muscle fiber

Table 1. Comparison of Blood Biochemistry Values of Dolphins, Horses, and Cows

\begin{tabular}{|c|c|c|c|}
\hline & Dolphins & Horses & Cows \\
\hline \hline Glucose (mg/dL) & $107.00 \pm 16.20$ & $100.67 \pm 4.84$ & $71.36 \pm 5.05^{*}$ \\
\hline TG (mg/dL) & $22.80 \pm 8.70$ & $14.33 \pm 5.28^{*}$ & $8.14 \pm 1.46^{*}$ \\
\hline T-Cho (mg/dL) & $195.20 \pm 61.20$ & $73.50 \pm 2.88^{*}$ & $217.07 \pm 40.57$ \\
\hline BUN (mg/dL) & $50.48 \pm 9.48$ & $17.40 \pm 3.17 *$ & $9.18 \pm 2.02^{*}$ \\
\hline Creatinine (mg/dL) & $1.22 \pm 0.23$ & $1.36 \pm 0.13$ & $0.60 \pm 0.09^{*}$ \\
\hline TP (g/dL) & $7.80 \pm 0.56$ & $6.23 \pm 0.340$ & $7.73 \pm 0.68$ \\
\hline LDH (IU/L) & $438.40 \pm 34.06$ & $216.67+58.84^{*}$ & $902.21 \pm 131.37 *$ \\
\hline AST (IU/L)) & $174.52 \pm 34.16$ & $275.05 \pm 33.25^{*}$ & $66.60 \pm 8.40^{*}$ \\
\hline ALT (IU/L) & $49.00 \pm 15.22$ & $6.50 \pm 1.64^{*}$ & $30.50 \pm 4.80^{*}$ \\
\hline ALP (IU/L) & $1344.80 \pm 661.55$ & $255.50 \pm 41.29^{*}$ & $146.64 \pm 78.94^{*}$ \\
\hline FFA (mEq/L) & $0.32 \pm 0.12$ & $0.04 \pm 0.02^{*}$ & $0.09 \pm 0.03^{*}$ \\
\hline
\end{tabular}

Values are presented as mean \pm SD

*Denotes significant difference as compared to dolphins (Mann-Whitney U-test, $\mathrm{p}<0.05$ ).

Table 2. Comparison of MDH and LDH Activities and M/L Ratio in Plasma of Dolphins, Horses and Cows

\begin{tabular}{|c|c|c|c|}
\hline & Dolphins & Horses & Cows \\
\hline \hline MDH & $134.51 \pm 90.17$ & $69.24 \pm 17.68$ & $90.00 \pm 20.69$ \\
\hline LDH & $199.00 \pm 27.63$ & $89.05 \pm 17.66^{*}$ & $604.21 \pm 184.58^{*}$ \\
\hline M/L ratio & $0.49 \pm 0.13$ & $0.79 \pm 0.15^{*}$ & $0.15 \pm 0.03^{*}$ \\
\hline
\end{tabular}

Values are presented as mean \pm SD.

*Denotes significant difference as compared to dolphins (Mann-Whitney U-test, $\mathrm{p}<0.05$ ). 
a)

\section{Dolphins}

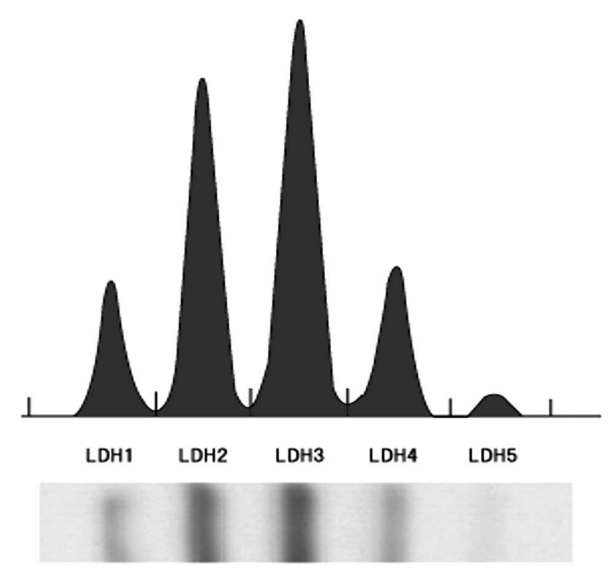

b)

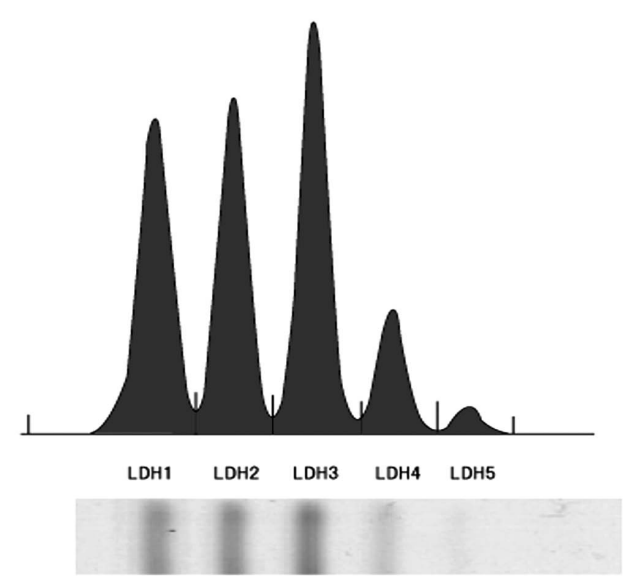

c)

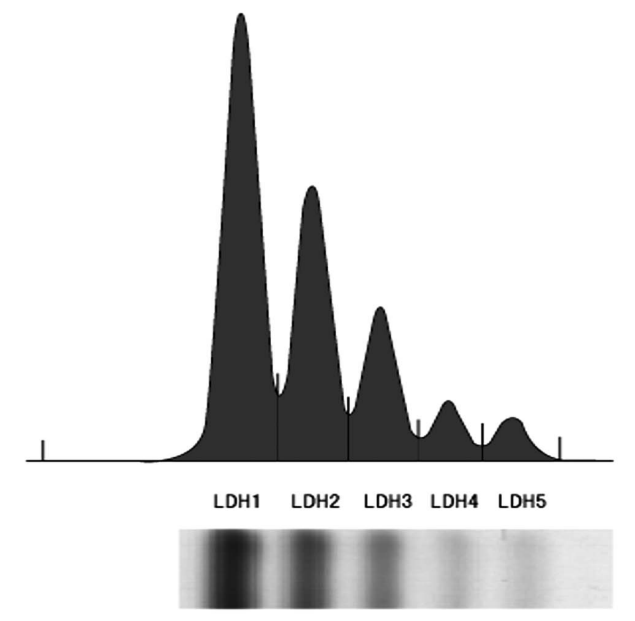

Fig. (1). Comparison of plasma LDH isoenzyme patterns between cows, horses, and dolphins. LDH isoenzymes were separated by an electrophoretic technique and analyzed using Epalyzer 2 Electrophoresis Processing Analyzer (Helena Laboratories). and metabolic profiles should reflect that species, as opposed to cows (do not run around at all) and horses (in between). Therefore, a difference in aerobic (endurance) $v s$ anaerobic muscle fiber profiles will have a great impact on ATP usage, in addition to general activity levels for example. Two fundamental reactions resynthesize ATP [14]: 1) Oxidative phosphorylation, breaking down carbohydrates, fats and protein, in the presence of oxygen, producing energy (ATP). The involvement of oxygen qualifies this as an aerobic reaction. 2) Glycolysis, breaking down glucose or glycogen into lactic acid. This reaction does not use oxygen and is considered anaerobic. There are several factors which will determine both the choice of fuel and the pathway used to generate ATP. These factors include: muscle fiber type, the speed and duration of exercise, type of feed provided, and animal fitness.

$\mathrm{M} / \mathrm{L}$ ratio appears to be one potential useful indicator of oxidative metabolism in animals since it can indirectly measure ATP synthesis/regeneration rate. A ratio of 1 would be ideal, since MDH enzyme activity to generate ATP is equally balanced by $\mathrm{LDH}$ enzyme activity to regenerate $\mathrm{NADH}$ into $\mathrm{NAD}^{+}$for additional energy production. Therefore, ATP is resynthesized at the same rate at which it is being used. However, since it is possible to obtain similar ratios, with differing $\mathrm{MDH}$ and $\mathrm{LDH}$ activity levels, it is imperative that $\mathrm{MDH}$ and $\mathrm{LDH}$ levels are also analyzed, in context, in order to reflect whether elevated energy metabolism, including elevated ATP production, may be occurring in some tissues, such as muscle and liver. Plasma $\mathrm{M} / \mathrm{L}$ ratio in horses $(0.79)$ was greater than that of dolphins (0.49) or cows (0.15); however $\mathrm{MDH}$ and $\mathrm{LDH}$ enzyme activity levels in dolphins was $\sim 1.9$ and $\sim 2.2 \mathrm{x}$ higher, respectively than that of horses; thereby indicating greater overall energy usage in dolphin than horse tissues. LDH, aldolase and phosphoglucomutase levels are higher in dolphin than in other mammalian skeletal muscles; pyruvate kinase is the only glycolytic enzyme in lower concentration in dolphin muscle [15] Therefore, although horses appear to use energy more efficiently than dolphins, there is greater energy usage or metabolism by dolphins as compared to horses, as evidenced in higher overall plasma MDH and LDH activity levels.

The horse has three basic types of muscle fiber: Type I, IIA and IIB, which differ in contractile and metabolic characteristics [14]. Type I fibers are slow-contracting fibers, while Types IIA and IIB are fast-contracting. In addition, Type I and IIA fibers have a high oxidative capacity and thus can utilize fuels aerobically while Type IIB fibers have a low aerobic capacity and depend on anaerobic glycolysis for energy generation. All three fiber types store glycogen, while only Types I and IIA have significant triglyceride storage. The speed of muscle contraction determines how fast the animal is able to move, and the faster an animal moves the greater the ATP requirement [14]. While walking, muscle contraction occurs very slowly and relatively small amounts of ATP are expended. During this type of exercise, Type I fibers are primarily recruited and energy generation is entirely aerobic, with muscle burning predominantly fat. As speed increases from a walk to a trot to a canter, Type I fibers alone are no longer capable of contracting rapidly enough to propel the horse. At this point, Type IIA fibers are also recruited. These fibers are also aerobic, but they use a 
Table 3. Comparison of LDH Isoenzyme Distribution Between Dolphins, Horses and Cows

\begin{tabular}{|c|c|c|c|}
\hline & Dolphins & Horses & Cows \\
\hline \hline LDH1 (\%) & $10.46 \pm 0.97$ & $29.88 \pm 3.02$ & $47.07 \pm 1.56$ \\
\hline LDH2 (\%) & $29.56 \pm 1.43$ & $25.30 \pm 2.63$ & $27.77 \pm 0.78$ \\
\hline LDH3 (\%) & $39.62 \pm 1.07$ & $31.55 \pm 2.20$ & $15.45 \pm 1.11$ \\
\hline LDH4 (\%) & $17.18 \pm 1.78$ & $10.67 \pm 0.99$ & $5.60 \pm 0.50$ \\
\hline LDH5 (\%) & $3.18 \pm 0.63$ & $2.60 \pm 0.95$ & $4.12 \pm 0.78$ \\
\hline
\end{tabular}

combination of glycogen and fat for energy generation. Glycogen (glucose) can be metabolized twice as fast as fat for ATP generation, and as speed increases, fat becomes simply too slow a fuel for energy generation. As the horse increases speed to a fast gallop, Type IIB fibers are recruited and energy generation no longer remains purely aerobic. At these speeds, the requirement for ATP has exceeded the ability of the horse to deliver enough oxygen to the muscle to produce the energy by aerobic means. Anaerobic glycolysis takes over as a rapid metabolic pathway to generate ATP. Anaerobic glycolysis results, however, in lactic acid accumulation, and fatigue soon develops as the $\mathrm{pH}$ in the muscle begins to fall.

Therefore, in the case of the riding horses used in our study, the majority of energy usage appears to be aerobic in origin due to the type of physical activity and muscle profile involved. These facts would support the greater plasma $\mathrm{M} / \mathrm{L}$ ratio in horses $(0.79)$ as opposed to dolphins (0.49) since $\mathrm{M} / \mathrm{L}$ ratio may be measuring oxidative ATP usage/regeneration by aerobic means only. Because dolphins are constantly moving, their energy usage may exceed that which can be produced by aerobic means only, and therefore $\mathrm{M} / \mathrm{L}$ ratio does not take ATP generation by anaerobic means into account.

To further support the idea of increased energy usage or metabolism in dolphins as opposed to horses, dolphins demonstrated significantly higher levels of plasma lipid metabolites, such as triglycerides ( $\mathrm{TG}, 0.5 \mathrm{x}$ greater), total cholesterol (T-Cho, 2.5x greater) and free fatty acid (FFA, $8 \mathrm{x}$ greater) as compared to horses. This may be indicative of dolphins having more capacity for lipolysis and lipid utilization as an additional energy source for aerobic ATP usage and regeneration, since they require more energy, and may have higher adipose stores as compared to horses [16, 17]. The difference in adipose stores between horses and dolphins may be possibly related to the type and quality of their diets [18-20]. In addition, seasonal variation of plasma $\mathrm{T}$-Cho and FFA levels in dolphins has been reported with TCho and FFA levels being inversely correlated with blubber thickness [21, 22], with FFA most likely being released from and deposited to the blubber in warm seasons and cooler winter months, respectively [22].

Additionally, dolphins exhibited the highest amount of plasma ALP amongst the animal species, being $\sim 5 \mathrm{x}$ and $\sim 9 \mathrm{x}$ greater than in horses and cows respectively. High levels of plasma ALP in dolphins have been previously observed in young and growing dolphins, with a significant decrease in concentration with increasing age [23].
Cows, on the other hand, demonstrated the lowest level of oxidative metabolism efficiency by aerobic means amongst all animal groups. Thus their aerobic energy usage may not be as efficient or may significantly differ from dolphins and horses, which would be expected according to "Symmorphosis" [8]. Since dolphins and horses are considered to be more active than cows, the increase in energy usage efficiency by aerobic means, of these 2 animal species could possibly be attributed to increased motility as compared to cows. In addition, since the cows are under a lactating state, energy usage by the animal is very different as opposed to a non-lactating state. Lactation in many species, particularly dairy cattle, which have been specifically bred for high milk production, requires a dramatic shift in metabolism redirecting a significant portion of ingested energy and metabolites to milk synthesis [24]. In cattle, hepatic gluconeogenesis provides the majority of glucose required for mammary lactose synthesis. The liver also breaks down nonesterified fatty acids (NEFA) as an additional energy source if there is insufficient acetate and volatile fatty acids (VFA) coming from digestion. In addition, glucose metabolism of muscle and adipose tissue is also modulated during lactation, with gluconeogenesis being down-regulated in muscle tissue, and a shift in the ratio of lipogenesis to lipolysis in adipose tissue, by hormones such as bovine somatotropin that stimulates galactopoiesis. In pregnant animals, these responses are exaggerated by moderate undernutrition and are mediated by reduced tissue sensitivity and responsiveness to insulin, associated with decreased tissue expression of the insulin-responsive facilitative glucose transporter, GLUT4. Peripheral tissue responses to insulin remain severely attenuated during early lactation but recover as the animal progresses through mid lactation. Therefore, the low level of energy metabolism, reflected by plasma $\mathrm{M} / \mathrm{L}$ ratio, observed in lactating cows is indicative of the high energy requirement redirected for lactation instead of to the animal.

Interestingly though, cows demonstrated the highest level of plasma LDH activity amongst all animal species, being $\sim 3 \mathrm{x}$ and $\sim 6.8 \mathrm{x}$ greater than dolphins and horses, respectively. Because lactating Holstein cows require more energy for lactation, LDH enzyme activity may be increased, in many tissues, as compared to non-lactating cows. In addition, rumen bacterial fermentation, due to lactate-producing organism such as Lactobacillus acidophilus could also affect LDH value in cows [29].

Different animal species exhibit differing characteristic plasma LDH isoenyzme patterns. In addition, each tissue has a characteristic composition of isoenzymes [25]. LDH is a 
tetrameric molecule made of four subunits of the parent molecules, and has five isoenzymes: LDH-1 (H4), LDH-2 (H3M1), LDH-3 (H2M2), LDH-4 (H1M3) and LDH-5 (M4) [25]. The present forms are termed $H$ (heart) and $M$ (muscle), for the organs from which they are obtained. Tissues containing a preponderance of LDH-1, with a lower $\mathrm{Km}$ value for pyruvate, usually function under aerobic conditions; whereas those containing a preponderance of LDH-5, function under relatively anaerobic conditions [26].

Although all animal groups displayed significant differences in isoenzyme pattern distribution, dolphins and horses demonstrated a similarity in plasma LDH isoenzyme pattern with LDH-3 predominating. However, the predominance patterns also reflect "Symmorphosis" of each species in that LDH-3,-2, and -4 predominated, in decreasing amount, in dolphins, which may often experience both anerobic and anaerobic energy utilization simultaneously, while LDH-1 and LDH-3 were equally predominant, with LDH-2 following in horses, which can also experience both aerobic and anaerobic energy utilization, however not simultaneously usually, and lastly LDH-1, -2 , and -3 were predominant in cows, which will tend to only experience aerobic energy utilization, due to their lack of general mobility. As such, $H$ type isoenzymes were relatively predominant in all of the animal species plasma examined in this study.

This study had several limitations. First, a small number of healthy dolphins and horses were available from the Enoshima Aquarium and the University Horse Riding club, respectively; therefore we had a limited number of animals in our study. Moreover, only one blood sample was drawn, per dolphin or horse, resulting in low statistical power, hence results and conclusions should be interpreted with care. Second, because the animals involved in the study were randomly selected, there was no age or gender matching performed. Aging can have an impact on energy consumption, however it is still uncertain whether it is influential enough to affect enzyme activities. For example, higher MDH activities, in skeletal muscle of older men, undergoing endurance training, have been reported [27]. Nonetheless, investigating for alternation in enzyme activities may be more appropriate if measured against age and gender matched animal groups. Three, postprandial influence might have had an impact on plasma metabolite values such as glucose and triglycerides. All blood samples, except from dolphins, were collected within $5 \mathrm{~h}$ postprandially, hence postprandial influence cannot be eliminated. In general, fasting is recommended before lipid screening because of the theoretical dynamic changes that can occur in test results for some lipid components, however this study focused on energy metabolism, therefore postprandial influence would be limited. Fourth, all animals involved in this study lived in captivity, and it is critical for this study to examine the animals in captivity. Particularly, blood samples collected from wild dolphins may affect the data by several reasons such as their handling stress and their diet [28]. Fifth, there was no standardized accounting or estimate of the activity and energy expenditure per day for the different animals used in our study. As such, no direct comparison could be made in terms of actual energy expenditure between the 3 different animal species.
In conclusion, dolphins appear to have the greatest level of oxidative energy metabolism amongst horses and cows, due to having the greatest levels of plasma MDH activity. In addition, dolphin energy production/usage efficiency was second $(\mathrm{M} / \mathrm{L}$ ratio $=0.49)$ behind that of horses $(\mathrm{M} / \mathrm{L}$ ratio $=$ 0.79). Overall, these results suggest that dolphins may possibly generate more energy than horses, especially for increased mobility in the water; however all of the produced energy is not utilized for some reasons, such as smaller tank size or other environmental limitations. However, cows demonstrated the highest plasma LDH activity amongst all animal species. This may have been attributed to their lactating state. Although all animal groups displayed a different plasma LDH isoenzyme pattern distribution, dolphins and horses demonstrated a similarity with LDH-3 isoenzyme predominating in plasma, as opposed to LDH-1 in cows, which would reflect "Symmorphosis" of these 2 species and their aerobic/anaerobic energy metabolism needs.

Overall, plasma MDH and LDH activity levels, M/L ratio, and plasma $\mathrm{LDH}$ isoenzyme pattern can all be useful indicators for better understanding oxidative energy metabolism and monitoring of captive animals' health. As it is not easy to obtain tissue samples from animals, the development of blood indicators for evaluating whole body metabolic state is necessary, and further research is required and should be pursued.

\section{ABBREVIATIONS}
ATP $=$ Adenosine triphosphate
$\mathrm{LDH}=$ Lactate dehydrogenase
$\mathrm{MDH}=$ Malate dehydrogenase
NAD $=$ Nicotinamide adenine dinucleotide
$\mathrm{NADH}=$ Nicotinamide adenine dinucleotide reduced form

\section{CONFLICT OF INTEREST}

The authors confirm that this article content has no conflict of interest.

\section{ACKNOWLEDGEMENTS}

The authors wish to thank Enoshima Aquarium and Koizumi Milk Farm for their help with collecting blood samples from animals and their valuable advice. This work was supported in part by the Strategic Research Base Development Program for Private Universities from the Ministry of Education, Culture, Sports, Science and Technology of Japan (MEXT), 2008-2012, and Grant-in-Aid for Scientific Research (No. 21380195) from the MEXT.

\section{REFERENCES}

[1] Arai T, Machida T, Sasaki M, Oki Y. Hepatic enzyme activities and plasma insulin concentrations in diabetic herbivorous voles. Vet Res Commun 1998; 13: 421-6.

[2] Arai T, Takahashi M, Araki K, Washizu T. Activities of enzymes related to the malate-aspartate shuttle in the blood cells of thoroughbreds undergoing training exercise. Vet Res Commun 2001; 25: 577-83.

[3] Setoyama C, Joh T, Tshuzuki T, Shimada K. Structural organization of the mouse cytosolic malate dehydrogenase gene: comparison with that of the mouse mitochondrial malate dehydrogenase gene. J Mol Biol 1988; 202: 355-64. 
[4] Hedeskov J, Capito K, Thams P. Cytosolic ratios of free $[\mathrm{NADPH}] /\left[\mathrm{NADP}^{+}\right]$and $[\mathrm{NADH}] /\left[\mathrm{NAD}^{+}\right]$in mouse pancreatic islets, and nutrient-induced insulin secretion. Biochem J 1987; 241: 161-7.

[5] Lauris V, Cahill GF. Hepatic glucose phosphotransferases. Variations among species. Diabetes 1966; 15: 475-9.

[6] Arai T, Inoue A, Uematsu Y, Sako T, Kimura N. Activities of enzymes in the malate-aspartate shuttle and the isoenzyme pattern of lactate dehydrogenase in plasma and peripheral leukocytes of lactating Holstein cows and riding horses. Res Vet Sci 2003; 75: $15-9$.

[7] Washizu T, Takahashi M, Azakami D, Ikeda M, Arai T. Activities of enzymes in the Malate-Aspartate shuttle in the peripheral leukocytes of dogs and cats. Vet Res Commun 2001; 25: 623-9.

[8] Taylor CR, Weibel ER. Design of the mammalian respiratory system. I. Problem and strategy. Respir Physiol 1981; 44: 1-10.

[9] Garland Jr T, Huey R. Testing symmophosis: Does structure match functional requirements? Evolution 1987; 41: 1404-9.

[10] Weibel ER, Taylor RC. Design of the mammalian respiratory system. Respir Physiol 1981; 44: 1-164.

[11] Shimamura M, Yasue H, Ohshima K, et al. Molecular evidence from retroposons that whales form a clade within even-toed ungulates. Nature 1997; 388: 666-70.

[12] Kimura N, Yoshimura I, Sako T, Inoue A, Tadami K, Arai T. Changes in activities of enzymes related to energy metabolism in peripheral leukocytes of fattening steers. Vet Res Commun 2005; 29: 19-26.

[13] Li G, Lee P, Mori N, Yamamoto I, Arai T. Long term intensive exercise training leads to a higher plasma malate/lactate dehydrogense $(\mathrm{M} / \mathrm{L})$ ratio and increased level of lipid mobilization in horses. Vet Res Commun 2012; 36(2): 149-55.

[14] Duren SE. Feeding the endurance horse. In: Advances in Equine Nutrition, Ed. Pagan, JD, Nottingham, UK: Nottingham University Press 1998; pp. 351-63.

[15] Storey KB, Hochachka PW. Glycolytic enzymes in muscle of the Pacific dolphin: role of pyruvate kinase in aerobic-anaerobic transition during diving. Comp Biochem Physiol B 1974; 49: 11928.

[16] Rommel SA, Lowenstine LJ. Gross and microscopic anatomy. In: Gulland FMD, Dierauf LA, Eds. CRC Handbook of Marine Mammal Medicine. $2^{\text {nd }}$ ed. Washington DC: CRC Press 2001; pp. $129-64$.

[17] Kasamatsu M, Kawauchi R, Tsunokawa M, et al. Comparison of serum lipid compositions, lipid peroxide, $\alpha$-tocopherol and lipoproteins in captive marine mammals (bottlenose dolphins, spotted seals and West Indian manatees) and terrestrial mammals. Res Vet Sci 2009; 86: 216-22.

[18] Asper ED, Cornell LH, Duffield DA, et al. Hematology and serum chemistry values in bottlenose dolphins. In: Leatherwood S, Reeves RR, Eds The Bottlenose Dolphin. New York, NY: Academic Press 1990; pp. 479-85.
[19] Bossart GD, Reidarson TH, Dierauf LA, Duffield DA. Clinical pathology. In: Gulland FMD, Dierauf LA. Eds. CRC Handbook of Marine Mammal Medicine, $2^{\text {nd }}$ ed. Washington, DC: CRC Press 2001; pp. 383-436.

[20] Kasamatsu M, Tsunokawa M, Taki M, Higuchi H, Nagahata H. Serum lipid peroxide and $\alpha$-tocopherol concentrations and superoxide dismutase activity in captive bottle-nosed dolphins. Am J Vet Res 2001; 62: 1952-6.

[21] Terasawa F, Kitamura M, Fujimoto A, Hayama S. Seasonal changes of blood composition in captive bottlenose dolphins. J Vet Med Sci 2002; 64: 1075-8.

[22] Williams TM, Haun JE, Friedl WA, Hall RW, Bivens LW. Assessing the thermal limits of bottlenose dolphins: a cooperative study by trainers, scientists and animals. IMATA Soundings Fall 1992: 16-17.

[23] Fair PA, Hulsey TC, Varela RA, et al. Hematology, serum chemistry, and cytology findings from apparently healthy Atlantic Bottlenose Dolphins (Tursiops truncatus) inhabiting the estuarine waters of Charleston, South Carolina. Aquat Mamm 2006; 32: 18295.

[24] Bell AW, Bauman DE. Adaptations of glucose metabolism during pregnancy and lactation. J Mammary Gland Biol Neoplasia 1997; 2: $265-78$.

[25] Dawson DM, Goodfriend TK, Kaplan NO. Lactic dehydrogenase: Functions of the two types. Science 1964; 143: 929-33.

[26] Latner AL, Siddiqui SA, Skillen AW. Pyruvate inhibition of lactate dehydrogenase activity in human tissue extracts. Science 1966; 154: 527-9.

[27] Suominen H, Heikkinen E. Enzyme activities in muscle and connective tissue of $M$. vastuslateralis in habitually training and sedentary 33 to 70-year-old men. Eur J Appl Physiol Occup Physiol 1975; 34: 249-54.

[28] Terasawa F, Kitamura M, Fujimoto A, Hayama S. Influence of diet on hematological characteristics in bottlenose dolphins. Jpn J Zoo Wildl Med 1999; 4: 117-24.

[29] Boyd J, West JW, Bernard JK. Effects of the addition of direct-fed microbials and glycerol to the diet of lactating dairy cows on milk yield and apparent efficiency of yield. J Dairy Sci 2011; 94: 461622.

[30] Terasawa F, Kitamura M. Hyperlipemia of captive bottlenose dolphins during pregnancy. J Vet Med Sci 2005; 67: 341-4.

[31] Kaloustian HD, Stolezenbach FE, Everse J, Kaplan NO. Lactate dehydrogenase of lobster (Hornarus americanus) tail muscle I. Physical and chemical properties. J Biol Chem 1969; 244: 2891901.

[32] Bergmeyer HU, Brent E. Malate dehydrogenase UV assay. In: Bergmeyer HU, Ed. Methods of enzymatic analysis, Vol.2. Verlag Chemie Weinheim, ed. New York, NY: Academic Press 1974; pp. 613-7. 